\title{
The medical case report
}

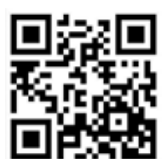

February's CME consists of a series of case reports that I have been sent over the past 12 months. Many journals local and international - feature case reports within their pages, and younger doctors in particular are encouraged to write up their more interesting cases in this format.

According to Wikipedia, ${ }^{[1]}$ 'In medicine, a case report is a detailed report of the symptoms, signs, diagnosis, treatment and follow-up of an individual patient'. Case reports are usually written to provide an unusual or a novel occurrence of a set of signs and symptoms, or, as is the case in some of the reports published this month, unusual presentations of a particular disease entity. Case reports generally contain a literature review of other reported cases, even if only to say that the report is of a rare occurrence.

Case reports are, by their very nature, anecdotal and placed at the foot of the hierarchy of clinical evidence, together with case series. However, case reports are usually thought to have a genuinely useful role in medical research and evidence-based medicine. A good example is the recognition of the link between giving thalidomide to pregnant women and malformations in their babies, which was triggered by a case report. Case reports have a role in pharmacovigilance and can contribute to the understanding of the clinical spectrum of rare diseases and unusual presentations in common ones. These anecdotal reports often help researchers to generate study hypotheses - including plausible mechanisms of disease.

However, one of the most useful roles of case reports is that of medical education, both formally, providing a structure for casebased learning (which we all did at medical school), and informally, for the general reader. In both cases, interesting and unusual presentations are helpful for day-to-day practice and often trigger recognition of a diagnosis or pathology in a puzzling clinical case.

All the case reports in this issue of CME are local, and I have tried to publish reports that will be particularly useful to our younger and less experienced colleagues. Please keep sending in your case reports. They are always welcome and interesting to read.

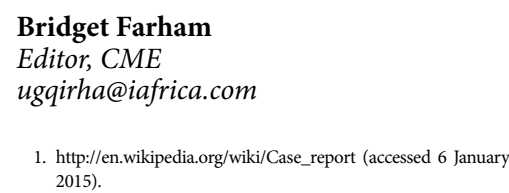

S Afr Med J 2015;105(2):151. DOI:10.7196/SAMJ.9350

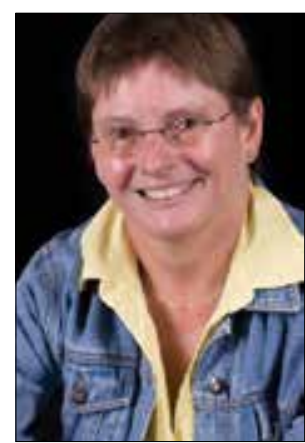

